\title{
Intramembranous Valine Linked to Schizophrenia Is Required for Neuregulin 1 Regulation of the Morphological Development of Cortical Neurons
}

\author{
Yachi Chen, ${ }^{1,3}$ Melissa L. Hancock, ${ }^{1,3,4}$ Lorna W. Role, ${ }^{1,3}$ and David A. Talmage ${ }^{2,3}$ \\ Departments of ${ }^{1}$ Neurobiology and Behavior and ${ }^{2}$ Pharmacological Sciences and ${ }^{3}$ Center for Nervous System Disorders, State University of New York, Stony \\ Brook, Stony Brook, New York 11794, and Department of Cell Biology, Harvard Medical School, Boston, Massachusetts 02115
}

\begin{abstract}
Neuregulin 1 (NRG1) signaling is critical to various aspects of neuronal development and function. Among different NRG1 isoforms, the type III isoforms of NRG1 are unique in their ability to signal via the intracellular domain after $\gamma$-secretase-dependent intramembranous processing. However, the functional consequences of type III NRG1 signaling via its intracellular domain are mostly unknown. In this study, we have identified mutations within type III NRG1 that disrupt intramembranous proteolytic processing and abolish intracellular domain signaling. In particular, substitutions at valine 321, previously linked to schizophrenia risks, result in NRG1 proteins that fail to undergo $\gamma$-secretase-mediated nuclear localization and transcriptional activation. Using processing-defective mutants of type III NRG1, we demonstrate that the intracellular domain signaling is specifically required for NRG1 regulation of the growth and branching of cortical dendrites but not axons. Consistent with the role of type III NRG1 signaling via the intracellular domain in the initial patterning of cortical dendrites, our findings from pharmacological and genetic studies indicate that type III NRG1 functions in dendritic development independent of ERBB kinase activity. Together, these results support the proposal that aberrant intramembranous processing and defective signaling via the intracellular domain of type III NRG1 impair a subset of NRG1 functions in cortical development and contribute to abnormal neuroconnectivity implicated in schizophrenia.
\end{abstract}

\section{Introduction}

The neuregulin 1 (NRG1) encodes a family of growth factors that interact with ERBB receptor tyrosine kinases via the epidermal growth factor (EGF)-like domain to initiate intracellular signaling cascades critical to neuronal functions (Kwon et al., 2005; Li et al., 2007; Woo et al., 2007; Mei and Xiong, 2008; Zhong et al., 2008; Barros et al., 2009; Fazzari et al., 2010; Wen et al., 2010). The NRG1 proteins are divided into six types, types I-VI. Most of NRG1 isoforms are single-pass transmembrane proproteins with the transmembrane domain (TMc) located at the $\mathrm{C}$ terminus. In contrast, type III NRG1 proproteins span the membrane twice because of the hydrophobic cysteine-rich domain (CRD). NRG1 proproteins undergo extracellular proteolytic processing at the

\footnotetext{
Received Feb. 3, 2010; revised April 30, 2010; accepted May 26, 2010.

This work was supported by grants from the National Alliance for Research on Schizophrenia and Depression/ Baer Foundation (Y.C., D.A.T., L.W.R.) and McKnight Brain Disorders (L.W.R.) and National Institutes of Health Grants DK07328 (M.L.H.) and NS29071 (L.W.R., D.A.T.). We thank Ping Ting Chen and Adnaan Sheriff for technical support and image analysis. Y.C. thanks David Lin for support and suggestions. Y.C. designed and performed all experiments in cortical neurons, analyzed data, and prepared this manuscript and figures. M.L.H. generated Gal $4_{D B D}$ fusion proteins containing wild-type and mutant forms of mouse type III NRG1, performed the initial alanine scanning mutagenesis, prepared B4-ECD proteins, and tested the specificity of anti-CRD antibody in the spinal cord. D.A.T. performed experiments testing for the effectiveness of the ERBB kinase inhibitor in T47D cells. D.A.T. and L.W.R provided valuable suggestions in the design of experiments and in data analysis and edited this manuscript.

The authors declare no competing financial interests.

Correspondence should be addressed to Yachi Chen, Department of Neurobiology and Behavior and Center for Nervous System Disorders, State University of New York, Stony Brook, Stony Brook, NY 11794. E-mail: yachchen@ms.cc.sunysb.edu.

D0I:10.1523/JNEUROSCI.0605-10.2010

Copyright $\odot 2010$ the authors $\quad 0270-6474 / 10 / 309199-10 \$ 15.00 / 0$
}

juxtamembrane region to release EGF-like domain, which then participates in paracrine signaling involving ERBB receptors (Falls, 2003). For type III NRG1, the cleaved ectodomain containing the EGF-like domain remains tethered to the membrane by the CRD. Thus, type III NRG1 is believed to signal via ERBB receptors in a juxtacrine manner (Wolpowitz et al., 2000; Leimeroth et al., 2002; Falls, 2003). Synaptic activation, membrane depolarization, or ERBB binding triggers a $\gamma$-secretase-dependent cleavage of type III NRG1 to release the intracellular domain (NRG1-ICD) from the membrane, which translocates to the nucleus to regulate gene expression (Bao et al., 2003, 2004). Type III NRG1-ERBB complexes therefore signal bidirectionally (i.e., via activation of ERBB and via the NRG1-ICD) (Wolpowitz et al., 2000; Leimeroth et al., 2002; Bao et al., 2003, 2004; Falls, 2003; Hancock et al., 2008; Zhong et al., 2008).

NRG1 is a candidate schizophrenia susceptibility gene (Stefansson et al., 2002). Studies of adult type III Nrg1 heterozygous mice have revealed defects reminiscent of schizophrenia-associated endophenotypes (Chen et al., 2008). One schizophrenia-associated NRG1 single-nucleotide polymorphism (SNP) changes a valine to leucine within the TMc (Walss-Bass et al., 2006). How this valine-to-leucine substitution affects schizophrenia susceptibility is unknown. We have begun addressing this issue by studying the effects of mutations within the TMc on the growth and branching pattern of cortical dendrites and axons because abnormal cortical connectivity has been implicated in schizophrenia (Rajkowska et al., 1998; Kalus et al., 2000). 
Here, we have shown differential requirements for type III NRG1-mediated signaling pathways in the development of dendrites versus axons of cortical neurons. Cortical neurons of type III Nrg1 knock-out (KO) embryos exhibit defects in the growth and branching of dendrites, a phenotype that is rescued by reexpression of wild-type (WT) type III NRG1 but not by NRG1 mutants that are defective in $\gamma$-secretase-dependent signaling, including the mutant containing the valine-to-leucine substitution within the TMc. Whereas NRG1 signaling via the intracellular domain plays a crucial role in dendritic development, NRG1 regulates axon extension via distinct mechanisms that do not involve intramembranous proteolytic cleavage and nuclear targeting. Together, our study provides evidence for differential requirements of NRG1 signaling in the development of cortical axons versus dendrites and insights into how the schizophreniaassociated valine-to-leucine mutation might contribute to the disease.

\section{Materials and Methods}

Primary cell cultures. Dispersed cortical neurons were prepared from C57BL/6 mice following previously described methods (Ghosh and Greenberg, 1995). Neuronal cultures were maintained in L-glutamine free basal medium Eagle media supplemented with 1\% N2 supplement, 1 $\mathrm{mm}$ L-glutamine, $5 \%$ fetal bovine serum, $100 \mathrm{U} / \mathrm{ml}$ penicillin, and 100 $\mu \mathrm{g} / \mathrm{ml}$ streptomycin. All reagents were purchased from Invitrogen.

Type III Nrg1 and ErbB4 mutant mice. We used wild-type and knockout littermates of a mouse line with genetic disruption of type III Nrg1 $\left(\mathrm{Nrg} 1^{\mathrm{tm} 1 / \mathrm{Lwr}}\right.$ ) (Wolpowitz et al., 2000). To label neurons, female type III $\mathrm{Nrg} 1$ heterozygous mice were bred with male transgenic mice overexpressing yellow fluorescent protein (YFP) from the Thy-1 promoter (line YFP-H) (Feng et al., 2000) (The Jackson Laboratory) in a subset of neurons. Animals from the resulting mouse line show YFP expression in the cortex. Male and female YFP-expressing, type III Nrg1 heterozygous mice were bred, and embryos generated from the crosses were used in the study. For studies involving ErbB4 knock-out animals, we used ErbB4 knock-out mice that were rescued from embryonic lethality by reexpression of ErbB4 in the heart (Tidcombe et al., 2003). The ErbB4 knock-out animals were kind gifts from Dr. G. Corfas (Harvard University, Boston, MA). The use of the animals was approved by the Institutional Animal Care and Use Committee of State University of New York, Stony Brook (Stony Brook, NY).

Generation of alanine substitutions. A $622 \mathrm{bp} \mathrm{BamHI}$ and HindIII fragment containing murine type III Nrg $\beta 1$ a sequences (position 378-1000; GenBank accession number AY648975) was subcloned into the pAlterExl vector (Promega). Mutant forms of type III Nrg1 were generated using the following primers and Altered Sites II In Vitro Mutagenesis System (Promega) according to the manufacturer's instruction: NRG1T307A/G308A, CTGACAATTGCTGCCATCTGTATC; NRG1-V321A/ V322A, ATCATGTGTGCGGCGGCCTACTGC; NRG1-K329A/Q330A, AAAACCAAGGCAGCGCGGCAGAAG. Inserts containing mutated sequences were sequenced and reinserted into full-length $\mathrm{Nrg} 1$ sequences using BamHI and HindIII sites. To generate $\mathrm{Gal}_{\mathrm{DBD}}$ fusion proteins, the Gal4 DNA binding domain was amplified by PCR from the pGBKT7 vector (BD Biosciences) using the following primers: forward, GGGAAGCTTATGAAGCTACTGTCTTCTATC; reverse, GGGAAGCTTCGATACAGTCAACTGTC. Gal4 ${ }_{\text {DBD }}$ was subcloned in frame into the full-length type III $\mathrm{Nrg \beta 1a}$ sequences at the HindIII site (position 1000). Type III Nrg $\beta 1 a-G a l 4_{\text {DBD }}$ was inserted into pSP72 $\beta$ act (Cho et al., 1998) under the transcription control of $\beta$-actin promoter.

Generation of valine-to-leucine substitution. Rat type III Nrgß1a sequences were cloned in pcDNA3.1 (D. L. Falls, Emory University, Atlanta, GA) (Wang et al., 2001). This plasmid was used as the template for mutagenesis. The valine residue at codon 322 of rat type III $\mathrm{Nrg \beta} 1 a$, which corresponds to valine 321 of murine type III Nrgß1a, was substituted to leucine by mutating the nucleic acid sequence from GTG to TTG (custom mutagenesis by Retrogen). This valine-to-leucine substitution was verified by DNA sequencing of the entire rat type III NrgB1a insert cloned into pcDNA3.1 (DNA sequencing by Retrogen). All codon numbering is based on murine type III NrgB1a sequences.

Preparation of soluble ERBB4 proteins. Media from HEK293 cells expressing the extracellular domain of ERBB4 fused to the human IgG Fc domain (B4-ECD) (Fitzpatrick et al., 1998) were concentrated using Microcon centrifugal filter devices (Millipore). B4-ECD fusion proteins were purified using protein A-agarose columns and detected by immunoblot analysis using an antibody specific for the human IgG Fc domain (Sigma-Aldrich). The integrity of the recombinant protein was confirmed by SDS-polyacrylamide gel separation. Dot blot analyses of purified ERBB4-ECD-Fc protein preparations with comparisons made to a dose curve of human IgG were performed; blots were probed with anti-Fc antibodies and quantified using a LI-COR Odyssey infrared imager to determine fusion protein levels. B4-ECD lacks the kinase domain. B4-ECD binds with high affinity to the EGF-like domain of NRG1 (Fitzpatrick et al., 1998) and has been used to induce NRG1 processing and NRG1-ICD release from the membrane (Bao et al., 2003).

Immunofluorescence studies. Cortical cultures were fixed in $4 \%$ formaldehyde/4\% sucrose/1× PBS for $15 \mathrm{~min}$. After fixation, cells were washed for 5 min with $1 \times$ PBS for three times. Subsequently, cells were blocked for $30 \mathrm{~min}$ with a blocking solution containing $10 \%$ normal donkey serum or $3 \%$ goat serum, depending on the secondary antibodies used, $0.25-0.3 \%$ Triton X-100, 3\% BSA, and $1 \times$ PBS. Cells were incubated at $4^{\circ} \mathrm{C}$ overnight with primary antibodies diluted in the blocking solution. Cells were washed three times and then incubated with secondary antibodies for $45 \mathrm{~min}$. All fluorophore-conjugated secondary antibodies were purchased from Invitrogen as $2 \mu \mathrm{g} / \mathrm{ml}$ stock solutions and used at a dilution of 1:1000. Cells were washed three times before mounting in ProlongGold (Invitrogen).

Subcellular localization of type III NRG1. For subcellular localization of type III NRG1, dispersed cortical neurons $\left(0.5 \times 10^{6}\right.$ cells per well $)$ were plated on 24-well plates containing $12 \mathrm{~mm}$ poly-lysine-laminin-coated glass coverslips (BD Biosciences). At the indicated day in vitro (DIV), cells were processed for immunofluorescence studies using sheep antiCRD [raised against a synthetic peptide derived from human type III NRG1 amino acid residues 92-122 (Yang et al., 1998); 1:1000; purified IgG] as the primary antibody and Alexa Fluor 488 donkey anti-sheep IgG as the secondary antibody. Images were captured with a Zeiss LSM 510 META scanning confocal microscope as $0.5 \mu \mathrm{m}$ stacks at $63 \times$ magnification. For colocalization with MAP2, the following primary and secondary antibody pairs were used: sheep anti-CRD and Alexa Fluor 568 donkey anti-sheep IgG; mouse anti-MAP2 ascites (1:3000; SigmaAldrich) and Alexa Fluor 488 donkey anti-mouse IgG. Neurons were counterstained with 4',6'-diamidino-2-phenylindole (DAPI) and imaged with a Zeiss LSM 510 multiphoton confocal microscope at $40 \times$ magnification, $1.5 \times$ digital zoom, and $0.5 \mu \mathrm{m}$ interval. For colocalization with VGLUT1 and MAP2, the following primary and secondary antibody pairs were used: sheep anti-CRD and Alexa Fluor 488 donkey anti-sheep IgG, mouse anti-MAP2 ascites and Alexa Fluor 647 donkey anti-mouse IgG, and rabbit anti-VGLUT1 (1:250; Synaptic Systems) and Alexa Fluor 568 donkey anti-rabbit IgG. Neurons were imaged with a Zeiss LSM 510 META confocal microscope at $100 \times$ magnification, $1.0 \times$ digital zoom, and $0.5 \mu \mathrm{m}$ interval.

Axon and dendrite studies. Dispersed embryonic day 18 (E18)/E19 cortical neurons $\left(0.5 \times 10^{6}\right.$ cells per well $)$ from wild-type and type III Nrg1 knock-out animals were plated on 24-well plates containing $12 \mathrm{~mm}$ polylysine-laminin-coated glass coverslips. A few hours after plating, cells were transfected with pEGFP-C3 encoding enhanced green fluorescent protein (EGFP) (0.15 $\mathrm{g}$ g/well; Clontech) using Lipofectamine 2000 (Invitrogen). For the rescue experiments, cortical neurons were transfected with pEGFP-C3 $(0.15 \mu \mathrm{g} /$ well $)$ alone or together with a plasmid $(0.45$ $\mu \mathrm{g} /$ well) encoding Gal4 ${ }_{\text {DBD }}$-tagged NRG1-WT, NRG1-T307A/G308A, NRG1-V321A/V322A, NRG1-K329A/Q330A or NRG1-WT or NRG1V321L with no Gal4 ${ }_{\mathrm{DBD}}$ tag. Two days after transfection, cells were processed for immunofluorescence studies. For ERBB kinase inhibitor experiments, cells were treated with DMSO (1:1000) or 4-[(3-bromophenyl)amino]-6(methylamino)-pyrido[3,4-d]pyridimine (PD158780) (1 $\mu \mathrm{M}$; Calbiochem) for $\sim 20-24 \mathrm{~h}$ before fixation. Transfected neurons were double labeled with rabbit anti-green fluorescent protein (GFP) antibodies (1:3000; In- 
vitrogen) to outline the entire neuron including processes and mouse anti-MAP2 antibodies (1:3000; Sigma-Aldrich) to identify the somatodendritic regions of neurons. The fluorophore-conjugated secondary antibodies used were Alexa Fluor 555 donkey anti-mouse IgG and Alexa Fluor 488 donkey anti-rabbit IgG. Cells were mounted in ProlongGold containing DAPI (Invitrogen).

Image stacks of GFP- and MAP2-positive neurons with intact nuclei showing no signs of nuclear fragmentation were captured at $40 \times$ magnification, $1.5 \times$ digital zoom, and $0.5 \mu \mathrm{m}$ interval using a Zeiss LSM 510 META scanning confocal microscope. Only neurons with identifiable axons were included in the analysis. Axons do not stain for MAP2 except in the region very proximal to the cell body. Axons were defined as the longest process extended from the cell body exhibiting a taper appearance and maintaining about the same thickness throughout the entire length. These features are reminiscent of those of neurons in the cortical plate (i.e., the cell dense zone just beneath the molecular layer) in vivo. Axons and dendrites were analyzed for the total length and number of branch points using Zeiss LSM software.

Studies of basal dendrites of YFP-labeled cortical neurons. Brains of E19 YFP-expressing, wild-type and type III Nrg1 heterozygous and knockout embryos were immersion fixed in $3 \%$ paraformaldehyde/ $1 \times$ PBS for $1 \mathrm{~h}$ at room temperature and sectioned into coronal sections $(300 \mu \mathrm{m})$ on a vibratome. Slices were immunostained with rabbit anti-GFP as the primary antibody and Alexa Fluor 488 donkey anti-rabbit IgG as the secondary antibody. Brain sections were mounted in ProlongGold. Image stacks of YFP-labeled neurons were captured at $40 \times$ magnification, $1.5 \times$ digital zoom, and $0.5 \mu \mathrm{m}$ interval using a Zeiss LSM 510 META scanning confocal microscope. Basal dendrites of individual neurons were traced through the entire image stack. Total length of all basal dendrites and number of basal dendritic branches of each neuron were quantified.

Luciferase reporter assays. Dispersed postnatal day $0(\mathrm{P} 0) / \mathrm{P} 1$ cortical neurons $\left(10^{6}\right.$ cells per well) from C57BL/6 mice were plated on polylysine-laminin-coated 12 -well plates and transfected at 2 DIV using Lipofectamine 2000. Cells were cotransfected with $0.5 \mu \mathrm{g}$ of constructs encoding mouse wild-type NRG1 (NRG1-WT) or different mutants of NRG1 fused with Gal4 ${ }_{\text {DBD }}$ (i.e., NRG1-T307A/G308A, NRG1-V321A/ V322A, or NRG1-K329A/Q330A) together with $0.5 \mu \mathrm{g}$ of Gal4-UASluciferase in p4Luc (Bao et al., 2003). To control for transfection efficiency, cells were also transfected with phRL-CMV (Promega) encoding Renilla luciferase. Twenty-four hours after transfection, cells were treated with $6.25 \mu \mathrm{l}$ per well of the vehicle ( $50 \%$ glycerol in $1 \times \mathrm{PBS})$ or $6.25 \mu \mathrm{l}$ per well of B4-ECD to a final concentration of $2 \mathrm{nM}$ under normal growth condition. Cells were harvested for luciferase assays $\sim 20 \mathrm{~h}$ after treatment. Luciferase assays were performed using Dual-Glo Luciferase Assay System (Promega) and a GloMax 96 Microplate Luminometer (Promega). Background values for firefly and Renilla luciferase were subtracted from all measurements. After background corrections, values of firefly luciferase were normalized against those of Renilla luciferase to control for transfection efficiency.

Nuclear localization studies of Gal4 immunoreactivity. Dispersed P0 cortical neurons $\left(0.6 \times 10^{6}\right.$ cells per well) from wild-type C57BL/6 mice were plated on 24 -well plates containing $12 \mathrm{~mm}$ poly-lysine-laminincoated glass coverslips. Cells were transfected at $1 \mathrm{DIV}$ with a plasmid (0.75 $\mu \mathrm{g} /$ well) encoding NRG1-WT or NRG1-V321A/V322A using Lipofectamine 2000. Two days after transfection, cells were treated with $10 \%$ DMSO in water (1:2000) or the $\gamma$-secretase inhibitor $(5 S)-(t$ butoxycarbonylamino)-6-phenyl-( $4 R$ )hydroxy-( $2 R$ ) benzylhexanoyl)L-leu-L-phe-amide (L685458) ( $1 \mu \mathrm{M}$; Sigma-Aldrich) for $1 \mathrm{~h}$ followed by a 20 min treatment with $3.75 \mu$ per well of $50 \%$ glycerol/1 $1 \times$ PBS or 3.75 $\mu \mathrm{l}$ per well of B4-ECD to a final concentration of $2 \mathrm{nM}$. Subsequently, cells were processed for immunofluorescence studies using the following primary and secondary antibody pairs: mouse anti-Gal4 (1:200; Abcam; or $2 \mu \mathrm{g} / \mathrm{ml}$; Zymed) together with Alexa Fluor 488 donkey anti-mouse IgG; sheep anti-CRD (1:500; purified IgG) together with Alexa Fluor 568 donkey anti-sheep IgG. Confocal image stacks were captured with a Zeiss LSM 510 multiphoton confocal microscope at $100 \times$ magnification and 1 $\mu \mathrm{m}$ interval. The single optical section through the nucleus of individual neuron was selected for analysis using NIH ImageJ. To determine the amount of nuclear Gal4 ${ }_{\mathrm{DBD}}$-tagged NRG1-ICD immunoreactivity, we measured the mean pixel value of Gal4 $4_{D B D}$ fluorescence intensity within the DAPI-stained nucleus. All measurements were normalized against those of vehicle-treated (i.e., $10 \%$ DMSO in water followed by $50 \%$ glycerol/1× PBS) NRG1-WT-expressing cells.

Statistical analysis. The results were analyzed by performing the nonparametric Mann-Whitney rank sum test for comparisons between two groups or Kruskal-Wallis test for multigroup comparisons using SigmaStat (SPSS). Statistically significant differences between control and experimental conditions are specified in the text.

\section{Results}

\section{Type III NRG1 regulates dendritic development of cortical} neurons in vivo

To investigate functions of type III NRG1 signaling in the development of neuroconnectivity in cortex, we began by studying the growth and branching of basal dendrites of cortical neurons from YFP-expressing, type III Nrg1 knock-out $\left(\mathrm{KO} / \mathrm{YFP}^{+}\right)$mice as well as their wild-type $\left(\mathrm{WT} / \mathrm{YFP}^{+}\right)$littermates. The expression of YFP from Thy1-YFP transgene has been shown to label only pyramidal neurons in the mouse cortex (Feng et al., 2000) (supplemental Fig. 1, available at www.jneurosci.org as supplemental material). In addition, the morphology of cortical pyramidal neurons is outlined by YFP localizing to cell bodies and projections (Wang et al., 2006). Confocal image stacks of YFP-labeled cortical pyramidal neurons were analyzed for the total length and number of branches of basal dendrites. We studied the basal den-

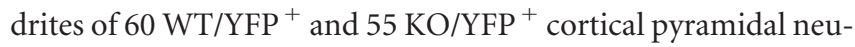
rons. Our results revealed that basal dendrites of cortical neurons from $\mathrm{KO} / \mathrm{YFP}^{+}$mice (i.e., $\mathrm{KO} / \mathrm{YFP}^{+}$neurons) showed a significant $52 \%$ reduction $(p<0.001)$ in the total length and a $44 \%$ decrease $(p<0.001)$ in the number of branches (Fig. 1). In contrast, $\mathrm{KO} / \mathrm{YFP}^{+}$mice show no apparent defect in the cortical plate thickness (supplemental Fig. 2, available at www.jneurosci. org as supplemental material), and this is consistent with normal development of cortical cell layers in mice lacking ERBB2/ERBB4 (Barros et al., 2009). In sum, type III NRG1 signaling is required for full elaboration of the basal dendritic arbors of cortical pyramidal neurons.

\section{Type III NRG1 function is essential for the elaboration of both dendrites and axons of cortical neurons in vitro}

To delineate the molecular signaling mechanisms involved in type III NRG1 function in the development of cortical connectivity, we performed additional experiments in cortical neuronal cultures. We began by examining the subcellular distribution of type III NRG1 in cortical neurons in culture using an antibody recognizing the CRD, which is present only in type III NRG1 (Yang et al., 1998) (supplemental Fig. 3, available at www. jneurosci.org as supplemental material). Type III NRG1 was detected throughout the soma and along both dendrites (MAP2positive processes) and glutamatergic axons (VGLUT1-positive but MAP2-negative processes) of cortical neurons (Fig. 2A-C). These results are consistent with the somatodendritic and presynaptic localization patterns of NRG1 isoforms in human prefrontal cortical neurons (Law et al., 2004) as well as type III NRG1 function in the development of cortical connectivity in vivo.

To study whether type III NRG1 regulates the growth and complexity of dendrites and axons of developing cortical neurons, we compared the total length and extent of branching of dendrites and axons of cortical neurons cultured in vitro from WT and type III Nrg1 KO mice. Cortical neurons were plated at equal densities and transfected with a plasmid encoding EGFP to outline the entire neuron including processes. Cells were immu- 
A WT/YFP+
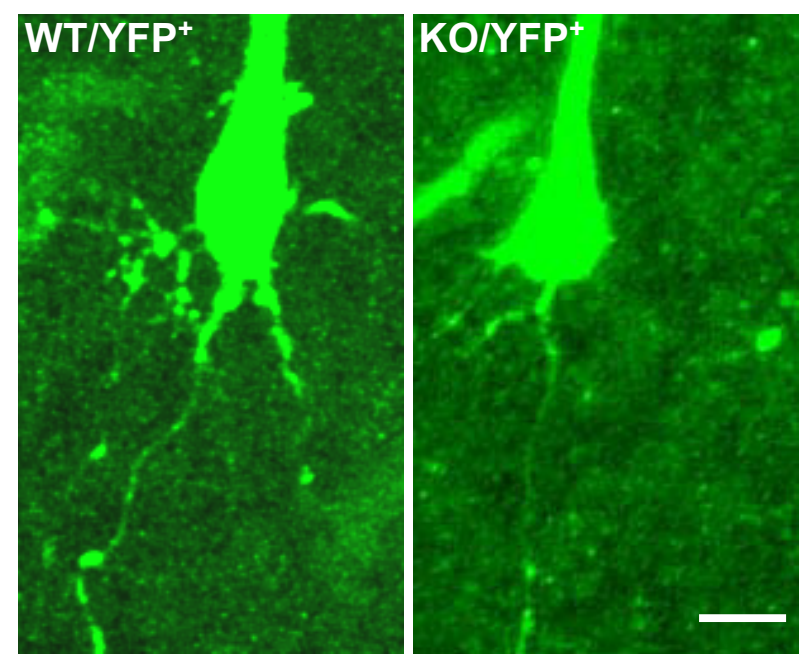

B

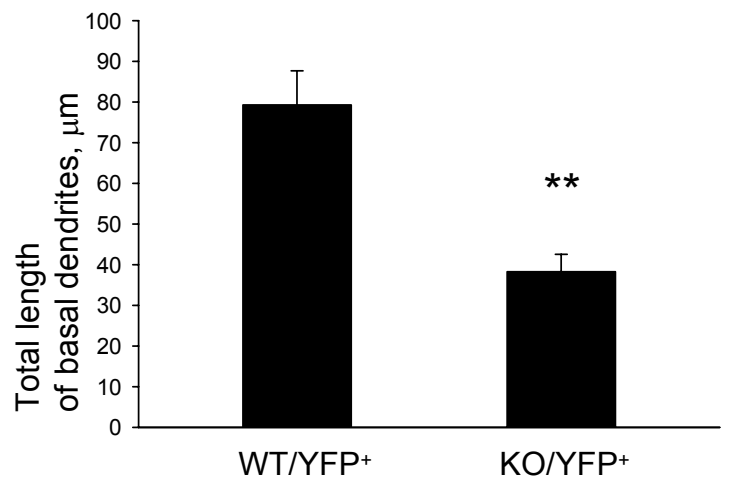

C

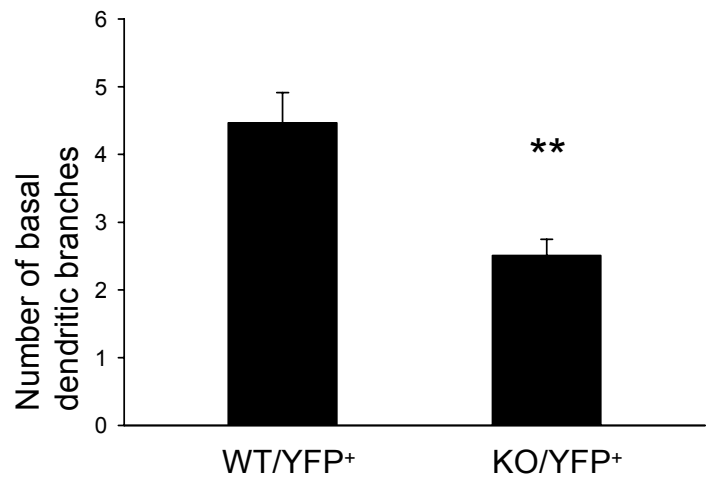

Figure 1. Type III NRG1 regulates dendritic development of cortical neurons in vivo. Confocal image stacks of YFP-labeled neurons in the cortical plate of E19 type III Nrg1 knock-out (KO/YFP ${ }^{+}$) mice as well as their wild-type (WT/YFP ${ }^{+}$) littermates were captured and analyzed for the total length and number of basal dendritic branches. $A$, Shown are images from collapsed image stacks of WT/YFP ${ }^{+}$and KO/YFP ${ }^{+}$cortical neurons. YFP expression is shown in green. KO/YFP ${ }^{+}$cortical neuron shows decreased complexity of basal dendrites. $\boldsymbol{B}, \boldsymbol{C}$, Results from KO/YFP ${ }^{+}$neurons were compared with that of WT/YFP ${ }^{+}$neurons. KO/YFP ${ }^{+}$neurons had reduced total basal dendritic length and fewer basal dendritic branches. The results are based on three independent experiments: WT/ $\mathrm{YFP}^{+}$neurons (60 neurons, 5 animals), KO/YFP ${ }^{+}$neurons (55 neurons, 3 animals). Scale bar, $10 \mu \mathrm{m}$. Values represent means \pm SEM. ${ }^{* *} p<0.001$.

nostained for EGFP and MAP2, a somatodendritic marker of neurons. Cortical neurons maintained in vitro are polarized with distinguishable dendrites versus axons (see Materials and Methods). Moreover, dendrites stain positive for MAP2, whereas axons do not stain for MAP2 except in the region very proximal to the cell body. Confocal image stacks of EGFP- and MAP2positive neurons were analyzed for the total length and number of branch points of dendrites and axons. This method of GFP labeling of the neuronal processes of mouse cortical neurons has been previously used to study a number of morphological parameters including total dendritic length and the number of branch points per cell (Whitford et al., 2002).

We quantified total dendritic length and the number of dendritic branch points for 47 WT cortical neurons and $64 \mathrm{KO}$ cortical neurons; $\mathrm{KO}$ neurons had a significant reduction in total dendritic length and number of branch points (length, $258.2 \pm$ 22.5 vs $130.9 \pm 9.8 \mu \mathrm{m}$; branch point, $7.5 \pm 1.3$ vs $3.4 \pm 0.5$ branch points; $p<0.05$ ) (Fig. $2 D-F)$. We also quantified axonal length and number of axonal branch points for $39 \mathrm{WT}$ cortical neurons and $88 \mathrm{KO}$ cortical neurons, and found that the total axonal length of $\mathrm{KO}$ neurons was significantly decreased $(668.2 \pm 72.7$ vs $443.2 \pm 36.5 \mu \mathrm{m} ; p<0.05)$ (Fig. $2 G)$. The number of axonal branch points was not significantly different between WT neurons versus KO neurons $(19.9 \pm 2.5$ vs $18.2 \pm$ 1.7 branch points) (Fig. $2 H$ ). Collectively, our results indicate that type III NRG1 signaling is critical to the elaboration of both dendrites and axons in the cortex.

\section{Alanine substitutions at residues $321 / 322$ in the C-terminal transmembrane domain abolish $\gamma$-secretase-dependent type III NRG1 signaling via the intracellular domain}

After synaptic activation, membrane depolarization or ERBB binding, type III NRG1 undergoes $\gamma$-secretase-dependent intramembranous cleavage to release the intracellular domain (NRG1-ICD) from the membrane (Bao et al., 2003, 2004) (Fig. $3 A)$. The released NRG1-ICD translocates to the nucleus to regulate gene expression (Bao et al., 2003, 2004) (Fig. 3A). To study whether NRG1 signaling via the intracellular domain contributes to the development of dendrites and axons, we began by identifying type III NRG1 mutants defective in processing and therefore signaling to the nucleus. We generated alanine mutations in the C-terminal transmembrane domain and the nuclear localization signal (NLS) of type III NRG1 (Fig. 3B). Subsequently, the ability of mutant forms of NRG1 to translocate to the nucleus was assayed by measuring transcriptional activation of a luciferase reporter gene in the nucleus. Cortical neurons were transiently cotransfected with constructs encoding full-length type III NRG1 (NRG1-WT) or one of three mutant forms of NRG1 (i.e., NRG1T307A/G308A, NRG1-V321A/V322A, and NRG1-K329A/Q330A) fused to Gal4 DNA-binding domain $\left(\mathrm{Gal}_{\mathrm{DBD}}\right)$, together with a luciferase reporter gene containing the Gal4-UAS promoter controlled by Gal4 ${ }_{\mathrm{DBD}}$. Under control conditions, neither wild-type nor mutant forms of NRG1 fused with Gal4 $4_{\mathrm{DBD}}$ induced the Gal4-UAS promoter and the transcription of the luciferase reporter (Fig. 3C). Previous studies demonstrated that treatment of cells with soluble ERBB4 containing only the extracellular domain fused to the Fc domain of human IgG (B4-ECD) (Fitzpatrick et al., 1998) leads to NRG1 proteolytic processing, nuclear translocation, and transcriptional activation (Bao et al., 2003). Treatment of NRG1-WT-expressing cells with 2 nM B4-ECD induced luciferase expression approximately threefold, indicating release and nuclear targeting of Gal4 ${ }_{\mathrm{DBD}}$-tagged NRG1-ICD ( $n=4$ vector, 6 NRG1-WT plus B4-ECD; $p<0.05$ ) (Fig. $3 C$ ). ERBB binding similarly induced luciferase expression in NRG1-T307A/G308Aexpressing neurons $(n=4$ NRG1-T307A/G308A plus B4-ECD; $p<0.05$ ) (Fig. 3C). Thus, mutations at residues $307 / 308$ had no effect on NRG1 processing, nuclear translocation, and transactivation. In contrast, in neurons expressing NRG1-V321A/V322A or NRG1-K329A/Q330A, the levels of luciferase activity after ERBB binding were similar to vector control $(n=5$ NRG1- 

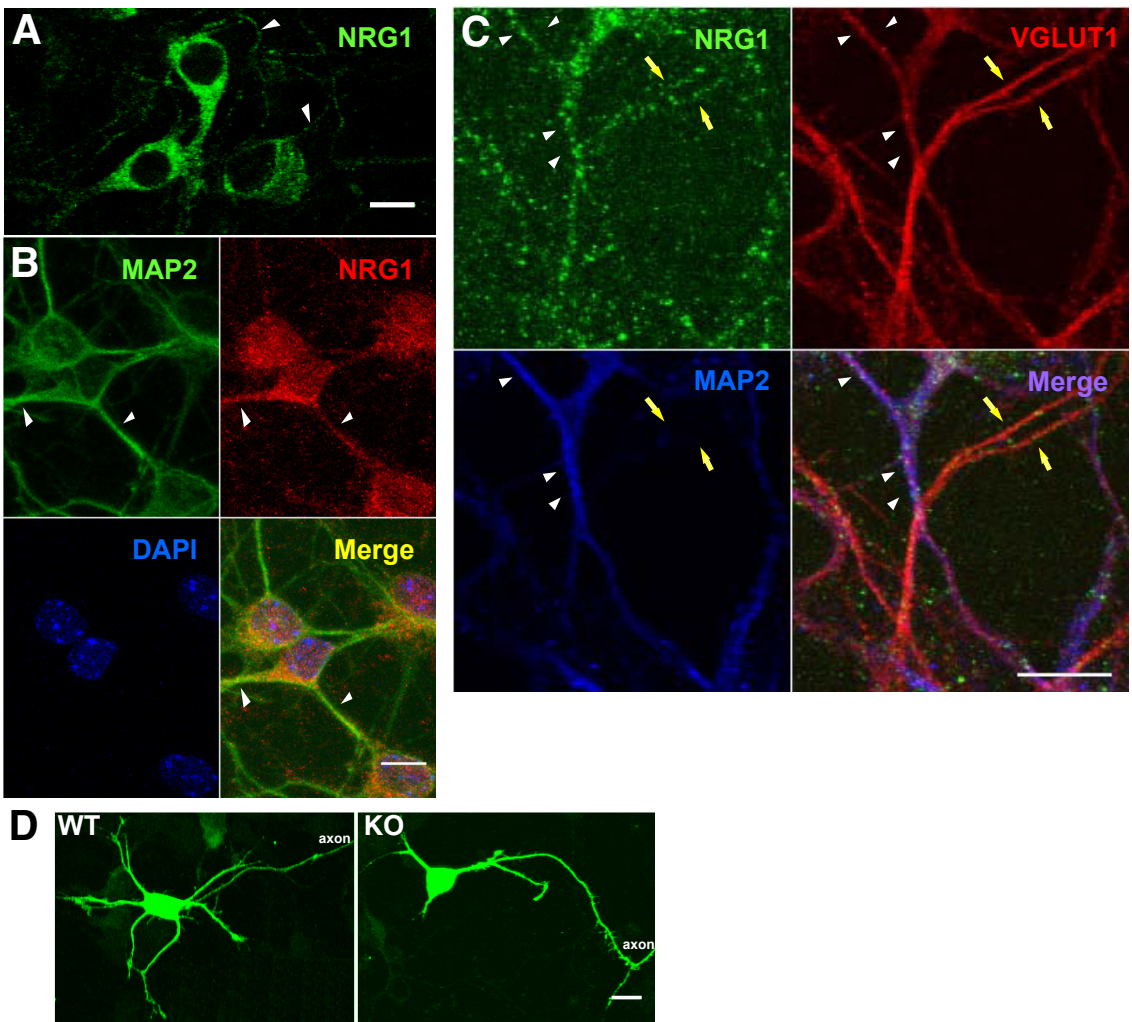

E

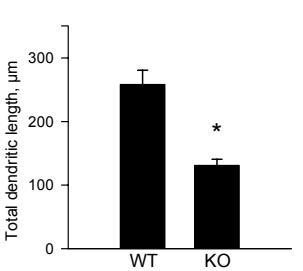

G
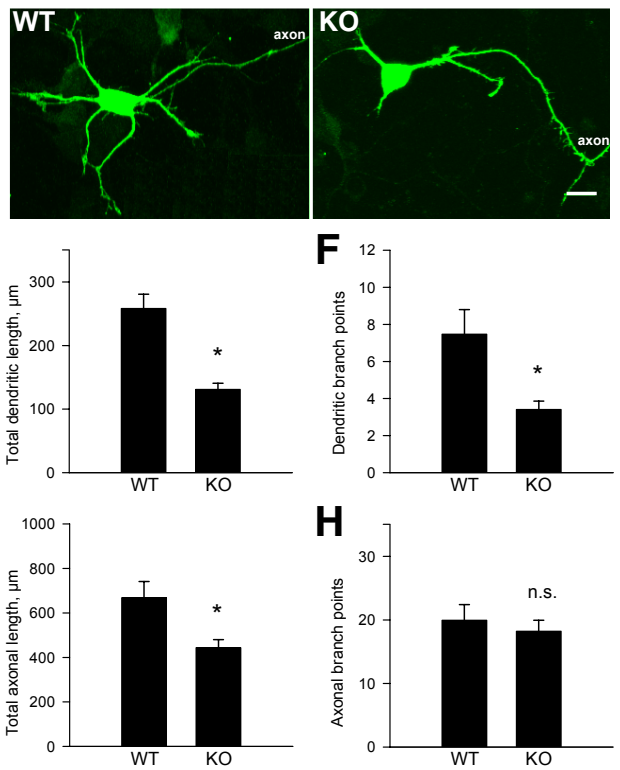

Figure 2. Type III NRG1 is expressed in cortical neurons and functions in the development of dendrites and axons in vitro. A, Cortical neurons (9 DIV) from E18 WT mice were processed for immunostaining of type III NRG1 (green). Endogenous type III NRG1 expression was detected in the cell bodies and neuronal processes (arrowheads). A single optical section through the center of the $z$-stack is shown. $\boldsymbol{B}$, Dispersed PO WT cortical neurons (3 DIV) were immunostained with antibodies for type III NRG1 (red) and the somatodendritic marker MAP2 (green). The nuclear morphology is revealed by DAPI staining (blue). Collapsed images of z-stacks are shown. Type III NRG1 is present in the cell bodies as well as MAP2-positive processes (arrowheads) of cells. C, Dispersed cortical neurons (3 DIV) from an E19 type III Nrg1 heterozygous mouse were immunostained with antibodies against type III NRG1 (green), MAP2 (blue), and the glutamatergic axonal marker VGLUT1 (red). Type III NRG1 was detected in the axonal branches (i.e., MAP2-negative but VGLUT1-positive processes; yellow arrows) and the dendritic branches (i.e., MAP2- and VGLUT1-positive processes; white arrowheads). Collapsed images of z-stacks are shown. D-H, EGFP (green) was transiently expressed in dispersedWT and KO neurons. Dendrites and axons of EGFP/MAP2 positiveneurons were analyzed for the total length and number of branch points. KO neurons showed shorter total dendritic length $(\boldsymbol{D}, \boldsymbol{E})$, fewer dendritic branch points $(\boldsymbol{D}, \boldsymbol{F})$, and shorter total axonal length $(\boldsymbol{G})$ than WT neurons. Axonal branch point numbers $(\boldsymbol{H})$ were not significantly different between WT versus KO neurons. Only partial view of the axon (i.e., the longest branch) is shown in $\boldsymbol{D}$. Note that the total length and number of branch points of dendrites or axons of WT versus KO neurons are also shown in Figure 4. See Figure 4 legend for additional information. Scale bars, $10 \mu \mathrm{m}$. Values represent means \pm SEM. ${ }^{*} p<0.05$; n.S., not significant.

V321A/V322A plus B4-ECD, 5 NRG1-K329A/Q330A plus B4ECD) (Fig. 3C), indicating that alanine substitutions at these residues interferes with $\mathrm{B} 4-\mathrm{ECD}$-induced NRG1 processing, nuclear targeting, and transcriptional activation.

To confirm that valine residues $321 / 322$ are required for $\gamma$-secretase-dependent type III NRG1 signaling in cortical neurons, we stimulated cortical neurons expressing a construct encoding NRG1-WT or NRG1-V321A/V322A with $\mathrm{B} 4-\mathrm{ECD}$ in the absence or presence of a $\gamma$-secretase inhibitor (L685458) and quantified immunoreactive Gal4 ${ }_{\mathrm{DBD}}$-tagged NRG1-ICD in the DAPI-labeled nucleus. The following results are from the analysis of nuclear Gal4 staining in a total of 101 cells from two independent experiments. In vehicle-treated NRG1-WT-expressing cells, Gal4 $4_{\mathrm{DBD}}$ (green) was detected in soma and processes and weakly in nuclei (blue), indicating low levels of nuclear targeting of NRG1-ICD in the basal state (Fig. 3D; each panel is from a single optical section through the center of individual nucleus). Stimulation of processing and translocation via B4-ECD treatment resulted in a 1.6-fold increase in nuclear Gal4 staining in NRG1-WT-expressing cells, indicating enhanced nuclear localization of $\mathrm{Gal}_{\mathrm{DBD}}$-tagged NRG1-ICD (Fig. 3D,E). This B4-ECD-stimulated NRG1-ICD nuclear localization in NRG1WT-expressing cells was blocked by inhibiting $\gamma$-secretase activity (Fig. 3E). This result is consistent with previously reported nuclear translocation of NRG1ICD mediated by $\gamma$-secretase in $293 \mathrm{~T}$ cells (Bao et al., 2003). However, B4-ECD failed to stimulate the nuclear translocation of NRG1-ICD in NRG1-V321A/ V322A-expressing cells (Fig. 3D,E). Moreover, the $\gamma$-secretase inhibitor had no additional effect on the nuclear level of NRG1-ICD in NRG1-V321A/V322Aexpressing cells, further implicating valine residues 321 and 322 in $\gamma$-secretase-mediated NRG1 processing (Fig. 3E). In sum, the valine residues at positions $321 / 322$ in the C-terminal transmembrane domain are essential for $\gamma$-secretase-dependent NRG1-ICD nuclear translocation and transcriptional activation.

\section{Rescue of defective dendritic}

phenotypes of type III Nrg1 knock-out cortical neurons by full-length type III NRG1 requires valine residues within the C-terminal transmembrane domain and nuclear localization signal

Defects in the complexity of dendrites and the outgrowth of axons of cortical neurons in type III Nrg1 mutant mice (Figs. 1, 2) indicated that aspects of type III NRG1 signaling contribute to the morphological development of cortical neurons. Type III NRG1-ERBB complexes can signal via activation of ERBB kinases and via the intracellular domain of NRG1 (Bao et al., 2003, 2004; Mei and Xiong, 2008). As such, we first tested whether NRG1 signaling via the intracellular domain contributes to the development of dendrites and axons. We analyzed the growth and branching pattern of 
dendrites and axons of type III Nrg1 KO neurons expressing NRG1-WT or NRG1 mutants defective in processing and intracellular domain signaling. $\mathrm{KO}$ neurons expressing NRG1-WT had total dendritic length and number of dendritic branch points that were similar to that of WT neurons and were significantly higher than those of $\mathrm{KO}$ neurons $(n=40$ NRG1-WT neurons) (Fig. 4A-C) (also see Fig. $4 D, E$ ) (35 NRG1-WT neurons; $p<$ $0.05)$. Thus, restoring type III NRG1 expression in $\mathrm{KO}$ neurons rescued dendritic defects in a cell-autonomous manner. Expression of NRG1-T307A/G308A restored dendritic length and branch points of KO neurons $(n=51$ NRG1-T307A/ G308A neurons; $p<0.05$ ) (Fig. $4 B, C$ ). In contrast, the total dendritic length and number of branch points of NRG1V321A/V322A-expressing KO neurons were comparable with that of $\mathrm{KO}$ neurons, indicating that the NRG1-V321A/ V322A mutant failed to rescue the dendritic defects of KO neurons $(n=43$ NRG1V321A/V322A neurons) (Fig. 4A-C). Therefore, the ability of type III NRG1 to restore normal dendritic phenotypes when expressed in $\mathrm{KO}$ neurons requires specific valine residues within the $\mathrm{C}$-terminal transmembrane domain, but not the nearby amino acids 307/308.

To directly demonstrate the importance of nuclear translocation of the NRG1-ICD in the regulation of dendritic development of cortical neurons, we assessed the effects of alanine substitutions of the NLS (Bao et al., $2003,2004)$ on the ability of NRG1 to restore normal dendritic phenotypes of $\mathrm{KO}$ neurons. As expected, full-length NRG1 expression restored the total dendritic length and branch points in $\mathrm{KO}$ neurons $(n=16$ WT neurons, $48 \mathrm{KO}$ neurons, 35 NRG1WT neurons; $p<0.05$ ) (Fig. $4 D, E$ ). However, we found that the total dendritic length and number of branch points of NRG1-K329A/Q330A-expressing KO neurons were comparable with that of $\mathrm{KO}$ neurons demonstrating that the NLS mutant, NRG1-K329A/Q330A, failed to rescue the dendritic defects of $\mathrm{KO}$ neurons ( $n=37$ NRG1-K329A/Q330A neurons) (Fig. 4D,E). Collectively, our results indicate that NRG1 mutants defective in $\gamma$-secretase-dependent signaling are unable to promote dendritic growth and branching of cortical neurons.

Type III NRG1 regulation of axonal growth of cortical neurons requires different aspects of NRG1 signaling Stimulated by our findings that the elaboration of dendrites requires type III NRG1
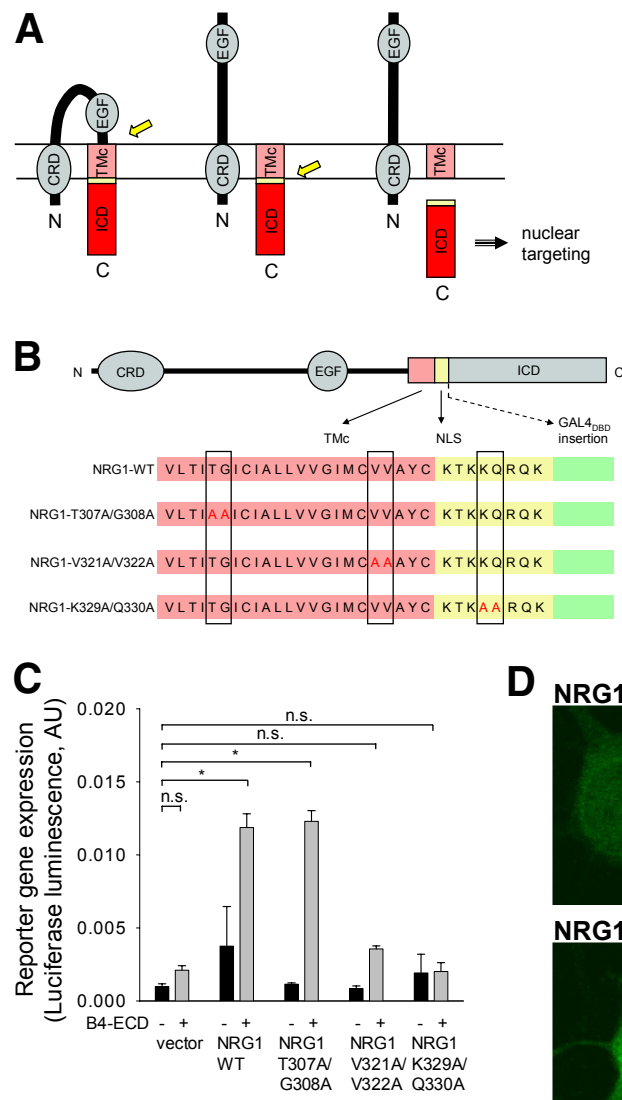

\section{NRG1-WT + vehicle}

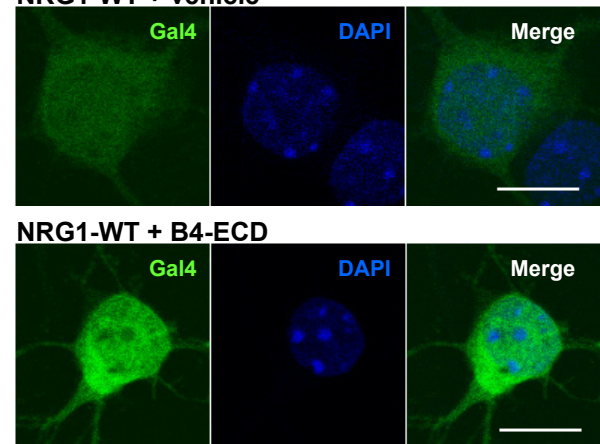

E

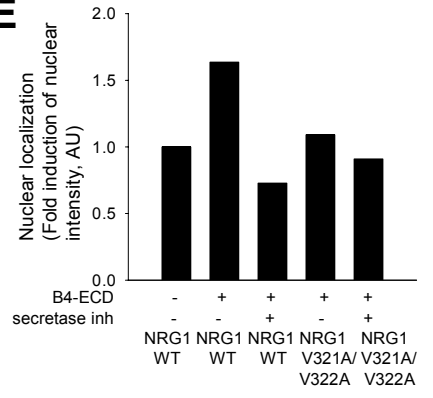

NRG1-V321A/V322A + B4-ECD

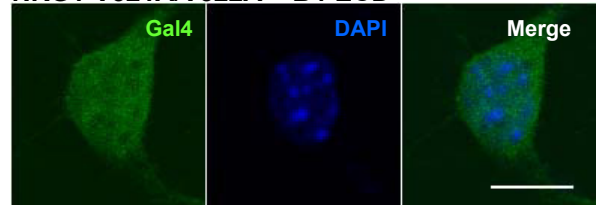

Figure 3. Valine residues 321 and 322 are involved in type III NRG1 intramembranous processing, nuclear translocation, and transactivation. $A$, Type III NRG1 undergoes proteolytic cleavage (yellow arrows), first in the extracellular juxtamembrane region and subsequently in the TMC. The second cleavage is $\gamma$-secretase dependent and releases NRG1-ICD from the membrane. N, N-terminal; C, C-terminal; CRD, cysteine-rich domain; EGF, EGF-like domain; TMc, C-terminal transmembrane domain; ICD, intracellular domain. $\boldsymbol{B}$, Schematic of chimeric wild-type NRG1 (NRG1-WT) and mutant NRG1 Gal4 $4_{D B D}$ fusion constructs used in the study. Positions of alanine substitutions are shown. NLS, Nuclear localization signal. C, NRG1-WT, NRG1-T307A/G308A, NRG1-V321A/N322A, or NRG1-K329A/Q330A Gal4 DBD $_{\text {Dimera was }}$ coexpressed with a luciferase reporter construct to assay nuclear localization and reporter gene expression in cortical neurons. Cells were treated with vehicle or the extracellular domain of ERBB4 (B4-ECD). Firefly/Renilla luciferase luminescence ratios were determined. Luminescence values (means \pm SEM) based on four individual experiments are shown: $n=4$ vector, 6 NRG1-WT plus B4-ECD, 4NRG1-T307A/ G308A plus B4-ECD, 5 NRG1-V321A/N322A plus B4-ECD, 5 NRG1-K329A/Q330A plus B4-ECD. AU, Arbitrary units. * $p<0.05$; n.s., not significant. D, E, NRG1-WT or NRG1-V321A/V322A was transiently expressed in cortical neurons. Cells were treated with vehicle or B4-ECD in the absence or presence of a $\gamma$-secretase inhibitor (L685458), processed for immunofluorescent localization of Gal $4_{D B D}$ (Gal4; green), and nuclear stained with DAPI (blue). Transfected neurons were identified based on Gal4 staining. Confocal image stacks of Gal ${ }_{D B D}$ localization were acquired. In D, a single optical section through the nucleus of a vehicle-treated NRG1-WT-expressing, a B4-ECD-treated NRG1-WTexpressing, or a B4-ECD-treated NRG1-V321A/N322A-expressing cell is shown in each panel. In vehicle-treated NRG1-WT-expressing cells, Gal4 immunoreactivity was detected in the soma and processes and weakly in nuclei. Treatment with B4-ECD enhanced nuclear Gal4 staining in NRG1-WT-expressing cells but not in NRG1-V321A/V322A-expressing cells. Nuclear Gal4 immunoreactivity was quantified in 30 and 71 neurons in two independent experiments, and results are presented in $E$. Within each experiment, the mean Gal 4 intensity from all analyzed neurons per condition was first determined and then normalized against that of vehicle-treated NRG1-WT-expressing cells to obtain "fold induction of nuclear Gal4 intensity." The average of two values of fold induction from two experiments is plotted in the graph. Stimulation of type III NRG1 processing and translocation via B4-ECD treatment resulted in 1.6-fold increase in the nuclear localization of $\mathrm{GaI}_{\mathrm{DBD}}$-tagged NRG1-ICD. In NRG1-WT-expressing cells, this increase in the nuclear localization of Gal ${ }_{\text {DBD }}$-tagged NRG1-ICD in response to B4-ECD was eliminated by the $\gamma$-secretase inhibitor. In NRG1-V321A/V322A-expressing cells, ERBB binding via B4-ECD treatment failed to stimulate nuclear translocation of Gal ${ }_{D B D}$-tagged NRG1-ICD in the absence or presence of the $\gamma$-secretase inhibitor. Scale bar, $10 \mu \mathrm{m}$. 
A

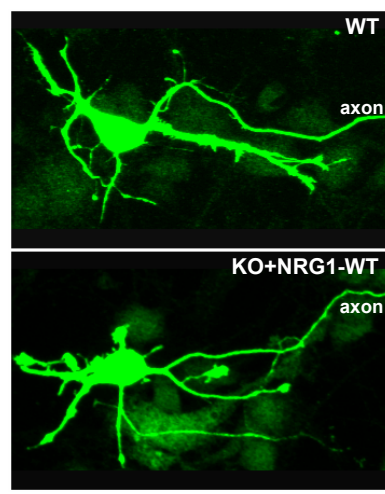

B

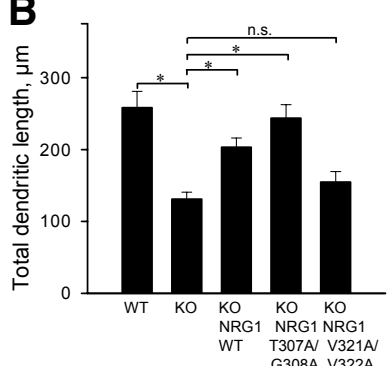

D

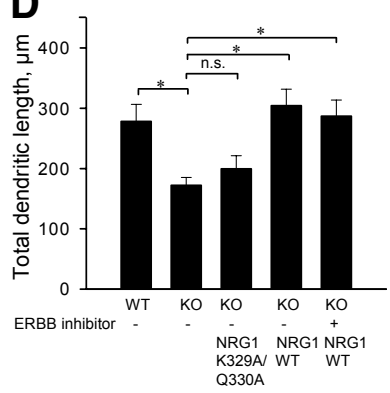

$\mathbf{F}$

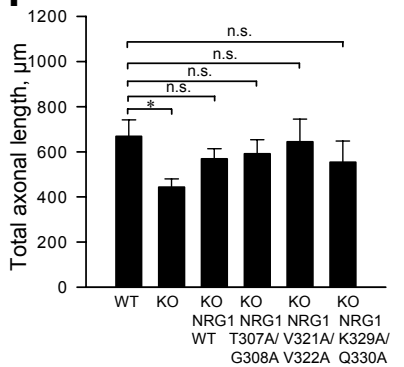

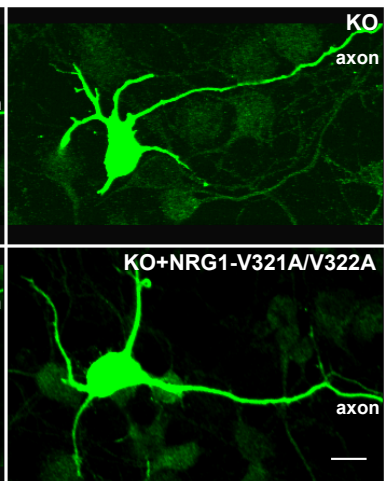

C

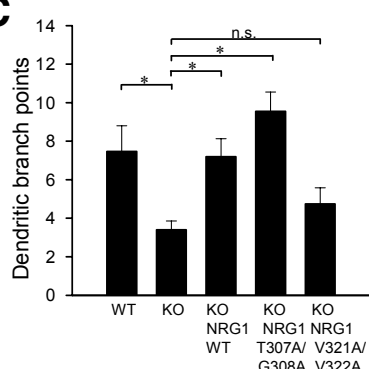

E

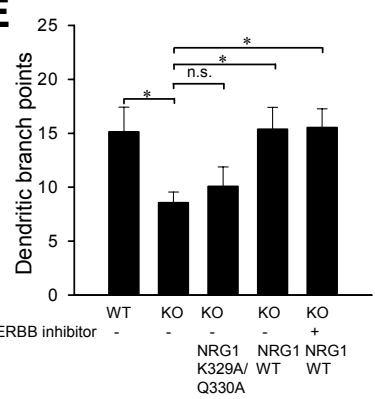

$\mathbf{G}$

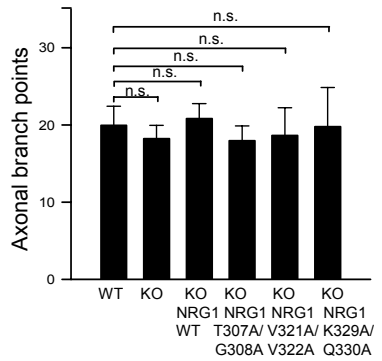

Figure 4. The development of cortical dendrites but not axons specifically requires nuclear targeting of the intracellular domain of type III NRG1. EGFP (green) was transiently expressed in dispersed WT and KO neurons. Rescue was assessed by coexpression of wild-type and mutant forms of NRG1 with EGFP. $A$, KO neurons show shorter dendritic length and fewer branch points than WT neurons. Coexpression of NRG1-WT in K0 neurons rescued dendritic growth and branching pattern. In contrast, coexpression of mutant NRG1-V321A/V322A in K0 neurons does not restore normal dendritic phenotypes. Only partial view of the axon (i.e., the longest branch) is shown. $\boldsymbol{B}-\boldsymbol{G}$, EGFP/MAP2-positive neurons were analyzed for total dendritic length $(\boldsymbol{B}, \boldsymbol{D})$, number of dendritic branch points $(\boldsymbol{C}, \boldsymbol{E})$, total axonal length $(\boldsymbol{F})$, and number of axonal branch points (G). Expression of NRG-WT or mutant NRG1-T307A/G308A in K0 neurons rescued dendritic length $(\boldsymbol{B}, \boldsymbol{D})$, number of dendritic branch points $(\boldsymbol{C}, \boldsymbol{E})$, and axonal length $(\boldsymbol{F})$. Mutant NRG1-V321A/V322A expression failed to restore the dendritic phenotypes of KO neurons ( $\boldsymbol{B}, \boldsymbol{C})$ but rescued the axonal length $(\boldsymbol{F})$. Similarly, expression of NRG1-K329A/Q330A in K0 neurons failed to rescue the total dendritic length $(\boldsymbol{D})$ and number of branch points $(\boldsymbol{E})$ but restored the axonal length $(\boldsymbol{F})$. WT neurons, KO neurons, and KO neurons expressing either wild-type or mutant forms of NRG1 exhibited comparable axonal branch points $(\boldsymbol{G})$. Where indicated in $\boldsymbol{D}$ and $\boldsymbol{E}$, NRG1-WT-expressing K0 neurons were treated with PD158780, an ERBB kinase inhibitor

signaling via the intracellular domain, we asked whether axonal growth also involves NRG1 signaling via the intracellular domain. To address this question, we assessed the ability of wildtype versus mutant forms of type III NRG1 to rescue the axonal phenotypes of $\mathrm{KO}$ neurons. In $\mathrm{KO}$ neurons expressing full-length type III NRG1, the total axonal length was similar to that of WT neurons demonstrating the ability of NRG1 to restore normal axonal growth of KO neurons ( $n=65$ NRG1-WT neurons) (Fig. $4 F)$. Furthermore, this ability of NRG1 to restore normal axonal growth was not affected by mutations of the transmembrane domain, or of the NLS; KO neurons expressing type III NRG1 mutants showed comparable total axonal length as WT neurons ( $n=45$ NRG1-T307A/G308A neurons, 34 NRG1-V321A/V322A neurons, and 17 NRG1-K329A/Q330A neurons) (Fig. 4F). Neither wild-type nor mutant forms of type III NRG1 affected the number of branch points of KO neurons (Fig. $4 G$ ). In contrast to dendritic development, type III NRG1 therefore regulates axonal growth in a cell-autonomous fashion that does not require the transcriptional functions of the NRG1-ICD.

\section{Rescue of dendritic morphology of cortical neurons is independent of ERBB kinase activity}

NRG1 binding to ERBB receptor kinases initiates intracellular signaling cascades that are critical to neuronal functions (Mei and Xiong, 2008). Previous studies have implicated NRG1-ERBB signaling in the morphological development of hippocampal neurons and dendritic development of cerebellar granule cells (Gerecke et al., 2004; Rieff and Corfas, 2006). In cortical neurons, it remains unclear whether NRG1 signaling via ERBB kinase activation contributes to the growth and branching of dendrites. We therefore assessed the ability of full-length type III NRG1 to restore normal dendritic phenotypes when expressed in $\mathrm{KO}$ neurons in the presence of an ERBB kinase inhibitor, PD158780. This inhibitor blocks ligand-induced activation of ERBB tyrosine kinases and prevents subsequent tyrosine phosphorylation of ERBB intracellular domain (supplemental Fig. 4, available at www.jneurosci.org as supplemental material). We found that the total dendritic length and number of branch points were comparable between untreated and ERBB kinase inhibitor-treated NRG1-WT-expressing KO neurons ( $n=48$ ERBB inhibitortreated NRG1-WT neurons) (Fig. 4D,E) (also see Fig. 5C,D) ( $n=30$ ERBB inhibitor-treated NRG1-WT neurons). Hence, the rescue of dendritic growth and branching by type III NRG1 does not require ERBB kinase activity.

(ERBB inhibitor). Expression of NRG1-WT in K0 neurons restored dendritic length $(\boldsymbol{D})$ and number of branch points $(\boldsymbol{E})$ even in the presence of the ERBB kinase inhibitor. The dendrite results presented in $\boldsymbol{B}$ and $\boldsymbol{C}$ are based on three independent transfections: WT neurons (47 neurons, 3 animals), K0 neurons (64 neurons, 5 animals), NRG1-WT-expressing K0 neurons (40 neurons, 3 animals), NRG1-T307A/G308A-expressing K0 neurons (51 neurons, 5 animals), and NRG1V321A/V322A-expressing K0 neurons (43 neurons, 4 animals). The dendrite data shown in $\boldsymbol{D}$ and $\boldsymbol{E}$ are based on two independent transfections: WT neurons (16 neurons, 2 animals), K0 neurons (48 neurons, 4 animals), NRG1-K329A/Q330A-expressing K0 neurons (37 neurons, 4 animals), NRG1-WT-expressing K0 neurons ( 35 neurons, 4 animals), and ERBB inhibitor-treated NRG1-WT-expressing KO neurons (48 neurons, 4 animals). The axon results presented in $\boldsymbol{F}$ and $G$ are based on four independent transfections: WT neurons ( 39 neurons, 3 animals), KO neurons (88 neurons, 6 animals), NRG1-WT-expressing K0 neurons (64 neurons, 6 animals), NRG1T307A/G308A-expressing K0 neurons (45 neurons, 5 animals), NRG1-V321A/V322Aexpressing KO neurons (34 neurons, 4 animals), and NRG1-K329A/Q330A-expressing K0 neurons (17 neurons, 2 animals). Scale bar, $10 \mu \mathrm{m}$. Values represent means \pm SEM. ${ }^{*} p<0.05$; n.s., not significant. 
A

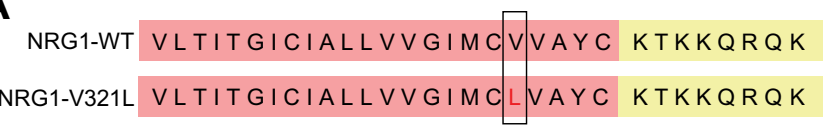

\section{B}
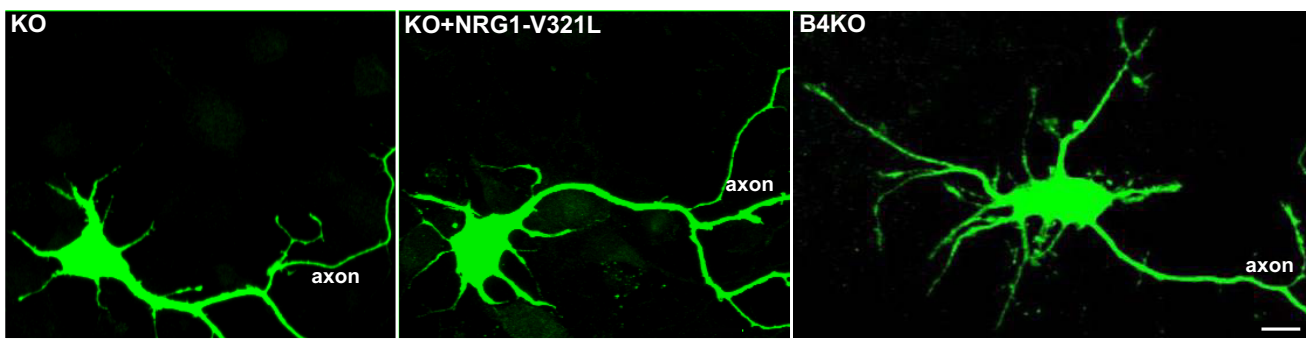

C

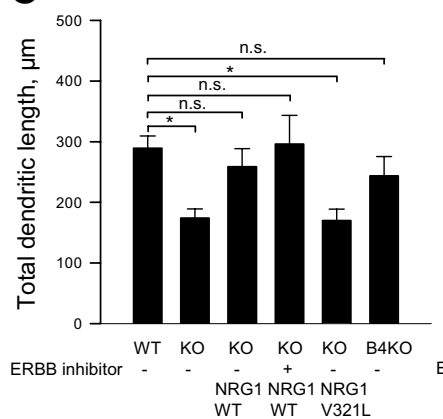

D

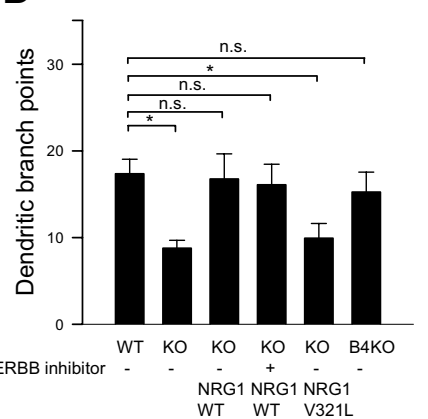

E

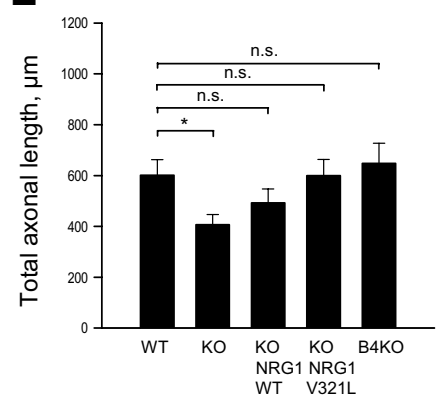

$\mathbf{F}$

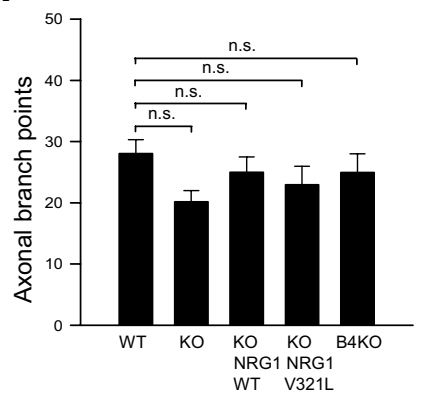

Figure 5. Type III NRG1 function in cortical dendritic development requires residue 321-mediated proteolytic processing and signaling via the intracellular domain and does not involve ERBB kinase activation. $\boldsymbol{A}$, Schematic of wild-type (NRG1-WT) and mutant form of type III NRG1 containing the valine-to-leucine substitution (NRG1-V321L). Note that both constructs do not contain the $\mathrm{Gal}_{\mathrm{DBD}}$ fusion protein. $\boldsymbol{B}-\boldsymbol{F}$, NRG1-WT or NRG1-V321L was coexpressed with EGFP in WT versus K0 cortical neurons. In $\boldsymbol{B}$, KO neuron and NRG1-V321L-expressing K0 neuron show comparable total dendritic growth and branching pattern. B4KO neuron from ErbB4 knock-out mouse shows more extensive dendritic development than K0 neuron from type III Nrg 1 knock-out animal. Only partial view of the axon (i.e., the longest branch) is shown. Expression of NRG1-WT but not NRG1-V321L in K0 neurons restored the total dendritic length ( $C$ ) and the number of dendritic branch points (D). The presence of an ERBB kinase inhibitor (ERBB inhibitor), PD158780, did not affect the ability of NRG1-WT to rescue dendritic phenotypes of K0 neurons ( $\boldsymbol{C}, \boldsymbol{D})$. The total dendritic length ( $\boldsymbol{C}$ ) and the number of dendritic branch points (D) of ErbB4 knock-out cortical neurons (B4K0) were not significantly different from that of WT neurons. Expression of NRG1-WT or NRG1-V321L in K0 neurons restored axonal length $(\boldsymbol{E})$. The total axonal lengths $(\boldsymbol{E})$ of WT neurons versus B4K0 neurons were comparable. WT neurons, K0 neurons, K0 neurons expressing either NRG1-WT or NRG1-V321L, and B4KO neurons were not significantly different in the number of axonal branch points $(\boldsymbol{F})$. The dendrite data shown in $\boldsymbol{C}$ and $\boldsymbol{D}$ are based on two independent transfections: WT neurons ( 42 neurons, 3 animals), K0 neurons (51 neurons, 4 animals), NRG1-WT-expressing KO neurons (29 neurons, 2 animals), ERBB inhibitor-treated NRG1-WT-expressing K0 neurons (30 neurons, 2 animals), NRG1-V321L-expressing KO neurons ( 34 neurons, 2 animals), and B4KO neurons ( 24 neurons, 2 animals). The axon results presented in $\boldsymbol{E}$ and $\boldsymbol{F}$ are based on three independent transfections: WT neurons ( 31 neurons, 2 animals), K0 neurons (54 neurons, 8 animals), NRG1-WT-expressing K0 neurons (27 neurons, 3 animals), NRG1-V321L-expressing K0 neurons ( 35 neurons, 3 animals), and B4K0 neurons (24 neurons, 2 animals). Scale bar, $10 \mu \mathrm{m}$. Values represent means \pm SEM. ${ }^{*} p<0.05 ;$ n.S., not significant.

\section{Cortical neurons of ErbB4 knock-out embryos show normal} development of dendrites and axons

The results of our pharmacological studies indicate that type III NRG1 might regulate the morphological development of cortical neurons independent of ERBB kinase activity in vivo. To test this possibility, we analyzed the growth and branching pattern of dendrites and axons of cortical neurons of ErbB 4 knock-out embryos rescued from early embryonic lethality by cardiac expression of ERBB4 (Tidcombe et al., 2003). The total dendritic length and number of dendritic branch points of ErbB4 knock-out cortical neurons (B4KO neurons) were not significantly different from that of WT neurons ( $n=24 \mathrm{~B} 4 \mathrm{KO}$ neurons) (Fig. $5 C, D)$. Our analysis also revealed that $\mathrm{B} 4 \mathrm{KO}$ neurons had normal axonal length and branch points ( $n=24 \mathrm{~B} 4 \mathrm{KO}$ neurons) (Fig. $5 E, F)$. Thus, the development of dendrites and axons of cortical neurons does not require ERBB4. Collectively, our results from pharmacological and genetic studies indicate that type III NRG1 signaling regulates growth and branching of cortical dendrites and axons in a cell-autonomous manner that does not require ERBB kinase activity.
The intramembranous valine residue 321 is required for type III NRG1 function in the development of dendrites but not axons of cortical neurons

Our results indicate that the ability of type III NRG1 to restore normal dendritic phenotypes of $\mathrm{KO}$ neurons requires specific valine residues within the $\mathrm{C}$-terminal transmembrane domain critical to intramembranous processing and nuclear translocation. A SNP that has been linked to increased risk for developing schizophrenia results in a leucine substitution for one of these valines (i.e., valine 321) (Walss-Bass et al., 2006). We therefore tested the effects of the comparable valine-to-leucine substitution of this residue (i.e., valine 321 ) on the ability of type III NRG1 to support normal dendritic development in $\mathrm{KO}$ neurons (Fig. 5A). As before, the expression of wild-type type III NRG1 in KO neurons restored the dendritic length and branch point number to levels of WT neurons ( $n=42$ WT neurons, $51 \mathrm{KO}$ neurons, 29 NRG1-WT neurons; $p<0.05$ ) (Fig. $5 C, D$ ). However, the total dendritic lengths and branch points were similar between $\mathrm{KO}$ neurons and KO neurons expressing the NRG1-V321L mutant, demonstrating that the mutant failed to rescue dendritic defects 
of KO neurons ( $n=34$ NRG1-V321L neurons) (Fig. $5 B-D$ ). Thus, the highly conserved valine residue in the C-terminal transmembrane region is critical to the ability of type III NRG1 to regulate dendritic phenotypes in cortical $\mathrm{KO}$ neurons.

We also assessed the ability of NRG1-V321L mutant to support normal axonal growth. As before, the expression of fulllength type III NRG1 in KO neurons restored total axonal length to levels comparable with that of WT neurons ( $n=31$ WT neurons, $54 \mathrm{KO}$ neurons, 27 NRG1-WT neurons) (Fig. 5E). NRG1V321L mutant also restored axonal growth $(n=35$ NRG1-V321L neurons) (Fig. 5E). In addition, WT neurons, KO neurons, and KO neurons expressing either NRG1-WT or NRG1-V321L are not significantly different in axonal branch points (Fig. $5 F$ ). We conclude that the highly conserved valine 321 involved in type III NRG1 signaling via the intracellular domain plays an important role in NRG1 regulation of dendritic development but is not required for NRG1 function in axonal growth.

\section{Discussion}

Our results reveal that type III NRG1 plays a crucial role in the normal development of both dendrites and axons of cortical neurons. Type III NRG1 regulation of dendritic complexity requires $\gamma$-secretase-dependent signaling via the NRG1 intracellular domain. Our pharmacological and genetic studies further indicate that type III NRG1 signaling via the intracellular domain regulates cortical dendritic development independent of ERBB kinase activity and, more specifically, ERBB4 function per se. These results not only reflect the ability of type III NRG1 to function as a receptor but also demonstrate the importance of type III NRG1 signaling via the intracellular domain in the development of cortical connectivity. In contrast to dendritic development, type III NRG1 regulation of axon extension does not involve $\gamma$-secretasedependent signaling via the intracellular domain and is likely to be related to $\gamma$-secretase-independent type III NRG1 activation of PtdIns 3-kinase signaling in axons (Hancock et al., 2008). In sum, our results clearly demonstrate differential requirements for type III NRG1-mediated signaling pathways in the growth and branching of dendrites versus axons in the developing cortex.

A substitution of valine residue 321 to leucine is associated with schizophrenia (Walss-Bass et al., 2006). In cells expressing NRG1 mutant containing this valine-to-leucine substitution as well as in cells deficient in $\gamma$-secretase, there is an increase in the levels of partially processed NRG1 (Dejaegere et al., 2008). These findings demonstrate that the valine mutation or a lack of $\gamma$-secretase results in ineffective NRG1 processing, thereby implicating a role of valine 321 in $\gamma$-secretase-mediated intramembranous cleavage. We show here that the valine substitution abolishes type III NRG1 signaling via the intracellular domain and leads to dendritic defects of cortical neurons presumably by disrupting $\gamma$-secretase-dependent intramembranous processing.

Type III NRG1 rescue of the dendritic defects of type III Nrg1 $\mathrm{KO}$ cortical neurons does not require ERBB kinase activity. In addition, cortical neurons from mice lacking ERBB4 do not have altered dendritic phenotypes. Therefore, type III NRG1 appears to regulate cortical dendritic development independent of ERBB kinase activation, and ERBB4 function per se. Clearly, our results in cortical pyramidal neurons are distinct from demonstrations for NRG1 function via ERBB kinase signaling in the morphological development of hippocampal neurons and cerebellar granule cells (Gerecke et al., 2004; Rieff and Corfas, 2006) as well as in promoting the maturation of dendritic spines (Barros et al., 2009). It remains unclear as to why NRG1 signaling via ERBB kinases plays a role in the morphological development of hip- pocampal neurons and cerebellar granule cells but not in cortical neurons. The differences in NRG1 versus ERBB requirement might reflect differences in their expression patterns. In the rodent hippocampus and cortex, ERBB4 expression has recently been shown to be selectively expressed in interneurons and not pyramidal neurons (Vullhorst et al., 2009; Fazzari et al., 2010; Neddens and Buonanno, 2010). In cerebellum, ErbB4 mRNA is expressed only in mature granule cells and not in other neuronal types (Elenius et al., 1997).

The lack of dendritic phenotypes in cortical neurons from the ErbB4 mutant embryos raises the question of how NRG1 signaling is stimulated. Synaptic activation and membrane depolarization have been shown to stimulate $\gamma$-secretase-dependent cleavage of type III NRG1 as well as other substrates (Bao et al., 2003, 2004; Inoue et al., 2009). There is mounting evidence for activity-dependent regulation of dendritic development of neurons (Chen and Ghosh, 2005; Flavell and Greenberg, 2008) and the presence of spontaneous activity in cultured cortical neuronal networks (Kamioka et al., 1996; Lin et al., 2002). Neuronal activity may thus represent a critical signal for triggering type III NRG1 proteolytic processing and signaling during cortical development.

In summary, we demonstrate that type III NRG1 plays an essential role in the development of cortical connectivity. The current study also uncovers a signaling mechanism for type III NRG1 that is specifically required for the development of dendrites but not axons of cortical neurons and provides insights into the ERBB kinase activity-independent mechanisms that control the early growth and patterning of cortical dendrites. Valine 321, which is required for NRG1 proteolytic cleavage, nuclear translocation, and function in dendritic development, is associated with increased risk for developing schizophrenia (Walss-Bass et al., 2006). This raises the intriguing possibility that altered NRG1 intramembranous processing and defective signaling via the intracellular domain of NRG1 contribute to abnormal development of neuroconnections implicated in the etiology of schizophrenia.

\section{References}

Bao J, Wolpowitz D, Role LW, Talmage DA (2003) Back signaling by the Nrg-1 intracellular domain. J Cell Biol 161:1133-1141.

Bao J, Lin H, Ouyang Y, Lei D, Osman A, Kim TW, Mei L, Dai P, Ohlemiller KK, Ambron RT (2004) Activity-dependent transcription regulation of PSD-95 by neuregulin-1 and Eos. Nat Neurosci 7:1250-1258.

Barros CS, Calabrese B, Chamero P, Roberts AJ, Korzus E, Lloyd K, Stowers L, Mayford M, Halpain S, Müller U (2009) Impaired maturation of dendritic spines without disorganization of cortical cell layers in mice lacking NRG1/ErbB signaling in the central nervous system. Proc Natl Acad Sci U S A 106:4507-4512.

Chen Y, Ghosh A (2005) Regulation of dendritic development by neuronal activity. J Neurobiol 64:4-10.

Chen YJ, Johnson MA, Lieberman MD, Goodchild RE, Schobel S, Lewandowski N, Rosoklija G, Liu RC, Gingrich JA, Small S, Moore H, Dwork AJ, Talmage DA, Role LW (2008) Type III neuregulin-1 is required for normal sensorimotor gating, memory-related behaviors, and corticostriatal circuit components. J Neurosci 28:6872-6883.

Cho Y, Klein MG, Talmage DA (1998) Distinct functions of protein kinase Calpha and protein kinase Cbeta during retinoic acid-induced differentiation of F9 cells. Cell Growth Differ 9:147-154.

Dejaegere T, Serneels L, Schäfer MK, Van Biervliet J, Horré K, Depboylu C, Alvarez-Fischer D, Herreman A, Willem M, Haass C, Höglinger GU, D'Hooge R, De Strooper B (2008) Deficiency of Aph1B/C- $\gamma$-secretase disturbs Nrg1 cleavage and sensorimotor gating that can be reversed with antipsychotic treatment. Proc Natl Acad Sci U S A 105:9775-9780.

Elenius K, Corfas G, Paul S, Choi CJ, Rio C, Plowman GD, Klagsbrun M (1997) A novel juxtamembrane domain isoform of HER4/ErbB4. Isoform-specific tissue distribution and differential processing in response to phorbol ester. J Biol Chem 272:26761-26768. 
Falls DL (2003) Neuregulins: functions, forms, and signaling strategies. Exp Cell Res 284:14-30.

Fazzari P, Paternain AV, Valiente M, Pla R, Luján R, Lloyd K, Lerma J, Marín O, Rico B (2010) Control of cortical GABA circuitry development by Nrg1 and ErbB4 signalling. Nature 464:1376-1380.

Feng G, Mellor RH, Bernstein M, Keller-Peck C, Nguyen QT, Wallace M, Nerbonne JM, Lichtman JW, Sanes JR (2000) Imaging neuronal subsets in transgenic mice expressing multiple spectral variants of GFP. Neuron 28:41-51.

Fitzpatrick VD, Pisacane PI, Vandlen RL, Sliwkowski MX (1998) Formation of a high affinity heregulin binding site using the soluble extracellular domains of ErbB2 with ErbB3 or ErbB4. FEBS Lett 431:102-106.

Flavell SW, Greenberg ME (2008) Signaling mechanisms linking neuronal activity to gene expression and plasticity of the nervous system. Annu Rev Neurosci 31:563-590.

Gerecke KM, Wyss JM, Carroll SL (2004) Neuregulin-1beta induces neurite extension and arborization in cultured hippocampal neurons. Mol Cell Neurosci 27:379-393.

Ghosh A, Greenberg ME (1995) Distinct roles for bFGF and NT-3 in the regulation of cortical neurogenesis. Neuron 15:89-103.

Hancock ML, Canetta SE, Role LW, Talmage DA (2008) Presynaptic type III neuregulin1-ErbB signaling targets $\alpha 7$ nicotinic acetylcholine receptors to axons. J Cell Biol 181:511-521.

Inoue E, Deguchi-Tawarada M, Togawa A, Matsui C, Arita K, KatahiraTayama S, Sato T, Yamauchi E, Oda Y, Takai Y (2009) Synaptic activity prompts gamma-secretase-mediated cleavage of EphA4 and dendritic spine formation. J Cell Biol 185:551-564.

Kalus P, Müller TJ, Zuschratter W, Senitz D (2000) The dendritic architecture of prefrontal pyramidal neurons in schizophrenic patients. Neuroreport 11:3621-3625.

Kamioka H, Maeda E, Jimbo Y, Robinson HP, Kawana A (1996) Spontaneous periodic synchronized bursting during formation of mature patterns of connections in cortical cultures. Neurosci Lett 206:109-112.

Kwon OB, Longart M, Vullhorst D, Hoffman DA, Buonanno A (2005) Neuregulin-1 reverses long-term potentiation at CA1 hippocampal synapses. J Neurosci 25:9378-9383.

Law AJ, Shannon Weickert C, Hyde TM, Kleinman JE, Harrison PJ (2004) Neuregulin-1 (NRG-1) mRNA and protein in the adult human brain. Neuroscience 127:125-136.

Leimeroth R, Lobsiger C, Lüssi A, Taylor V, Suter U, Sommer L (2002) Membrane-bound neuregulin1 type III actively promotes Schwann cell differentiation of multipotent progenitor cells. Dev Biol 246:245-258.

Li B, Woo RS, Mei L, Malinow R (2007) The neuregulin-1 receptor erbB4 controls glutamatergic synapse maturation and plasticity. Neuron 54:583-597.

Lin YC, Huang ZH, Jan IS, Yeh CC, Wu HJ, Chou YC, Chang YC (2002) Development of excitatory synapses in cultured neurons dissociated from the cortices of rat embryos and rat pups at birth. J Neurosci Res 67:484-493.

Mei L, Xiong WC (2008) Neuregulin 1 in neural development, synaptic plasticity and schizophrenia. Nat Rev Neurosci 9:437-452.

Neddens J, Buonanno A (2010) Selective populations of hippocampal inter- neurons express ErbB4 and their number and distribution is altered in ErbB4 knockout mice. Hippocampus 20:724-744.

Rajkowska G, Selemon LD, Goldman-Rakic PS (1998) Neuronal and glial somal size in the prefrontal cortex: a postmortem morphometric study of schizophrenia and Huntington disease. Arch Gen Psychiatry 55:215-224.

Rieff HI, Corfas G (2006) ErbB receptor signalling regulates dendrite formation in mouse cerebellar granule cells in vivo. Eur J Neurosci 23:2225-2229.

Stefansson H, Sigurdsson E, Steinthorsdottir V, Bjornsdottir S, Sigmundsson T, Ghosh S, Brynjolfsson J, Gunnarsdottir S, Ivarsson O, Chou TT, Hjaltason O, Birgisdottir B, Jonsson H, Gudnadottir VG, Gudmundsdottir E, Bjornsson A, Ingvarsson B, Ingason A, Sigfusson S, Hardardottir H, et al. (2002) Neuregulin 1 and susceptibility to schizophrenia. Am J Hum Genet 71:877-892.

Tidcombe H, Jackson-Fisher A, Mathers K, Stern DF, Gassmann M, Golding JP (2003) Neural and mammary gland defects in ErbB4 knockout mice genetically rescued from embryonic lethality. Proc Natl Acad Sci U S A 100:8281-8286.

Vullhorst D, Neddens J, Karavanova I, Tricoire L, Petralia RS, McBain CJ, Buonanno A (2009) Selective expression of ErbB4 in interneurons, but not pyramidal cells, of the rodent hippocampus. J Neurosci 29:1225512264.

Walss-Bass C, Liu W, Lew DF, Villegas R, Montero P, Dassori A, Leach RJ, Almasy L, Escamilla M, Raventos H (2006) A novel missense mutation in the transmembrane domain of neuregulin 1 is associated with schizophrenia. Biol Psychiatry 60:548-553.

Wang JY, Miller SJ, Falls DL (2001) The N-terminal region of neuregulin isoforms determines the accumulation of cell surface and released neuregulin ectodomain. J Biol Chem 276:2841-2851.

Wang Y, Zhang J, Mori S, Nathans J (2006) Axonal growth and guidance defects in Frizzled3 knock-out mice: a comparison of diffusion tensor magnetic resonance imaging, neurofilament staining, and genetically directed cell labeling. J Neurosci 26:355-364.

Wen L, Lu YS, Zhu XH, Li XM, Woo RS, Chen YJ, Yin DM, Lai C, Terry AV Jr, Vazdarjanova A, Xiong WC, Mei L (2010) Neuregulin 1 regulates pyramidal neuron activity via ErbB4 in parvalbumin-positive interneurons. Proc Natl Acad Sci U S A 107:1211-1216.

Whitford KL, Marillat V, Stein E, Goodman CS, Tessier-Lavigne M, Chédotal A, Ghosh A (2002) Regulation of cortical dendrite development by SlitRobo interactions. Neuron 33:47-61.

Wolpowitz D, Mason TB, Dietrich P, Mendelsohn M, Talmage DA, Role LW (2000) Cysteine-rich domain isoforms of the neuregulin-1 gene are required for maintenance of peripheral synapses. Neuron 25:79-91.

Woo RS, Li XM, Tao Y, Carpenter-Hyland E, Huang YZ, Weber J, Neiswender H, Dong XP, Wu J, Gassmann M, Lai C, Xiong WC, Gao TM, Mei L (2007) Neuregulin-1 enhances depolarization-induced GABA release. Neuron 54:599-610.

Yang X, Kuo Y, Devay P, Yu C, Role L (1998) A cysteine-rich isoform of neuregulin controls the level of expression of neuronal nicotinic receptor channels during synaptogenesis. Neuron 20:255-270.

Zhong C, Du C, Hancock M, Mertz M, Talmage DA, Role LW (2008) Presynaptic type III neuregulin 1 is required for sustained enhancement of hippocampal transmission by nicotine and for axonal targeting of $\alpha 7$ nicotinic acetylcholine receptors. J Neurosci 28:9111-9116. 